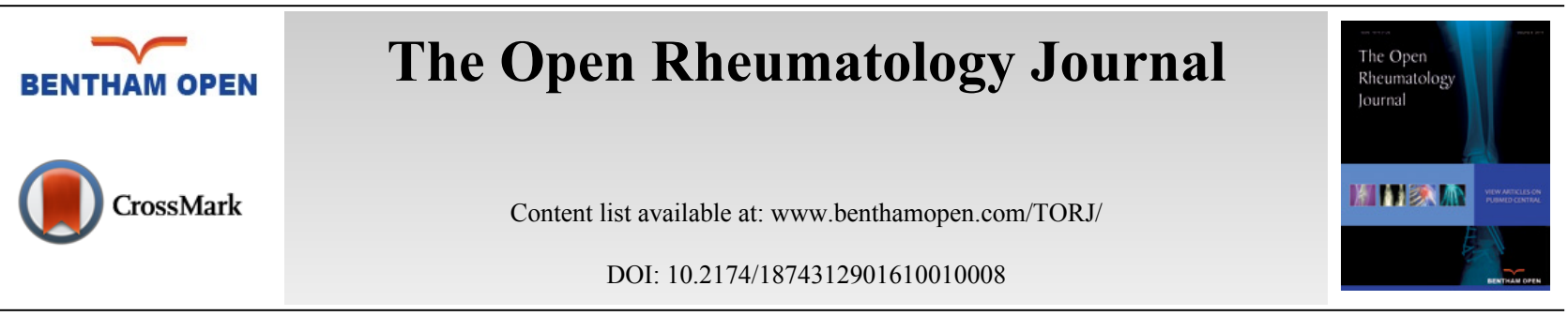

\title{
An Open Randomized Trial Comparing the Effects of Oral NSAIDs Versus Steroid Intra-Articular Infiltration in Congestive Osteoarthritis of the Knee
}

Ouédraogo Dieu-Donné ${ }^{1, *}$, Ouédraogo Théodore ${ }^{2}, Z^{2}$ absonré/Tiendrébéogo Joëlle ${ }^{1}$, Dionou Pierre ${ }^{1}$, Ouédraogo Smaïla ${ }^{3}$, Compaoré Christian ${ }^{4}$, Kaboré Fulgence ${ }^{1}$ and Drabo Youssouf Joseph ${ }^{5}$

${ }^{I}$ Department of Rheumatology, University Hospital Yalgado Ouedraogo, Ouagadougou, Burkina Faso

${ }^{2}$ Department of Anatomy; UFR SDS, University of Ouagadougou, Burkina Faso

${ }^{3}$ Department of Epidemiology and Statistic, University Hospital Yalgado Ouedraogo, Ouagadougou, Burkina Faso

${ }^{4}$ Department of Internal Medicine, International Polyclinic, Ouagadougou, Burkina Faso

${ }^{5}$ Department of Internal Medicine, University Hospital Yalgado Ouedraogo, Ouagadougou, Burkina Faso

\begin{abstract}
:
Objective:

To compare the efficacy on pain and joint function of NSAIDs versus steroid intra-articular infiltration in congestive knee osteoarthritis.
\end{abstract}

\section{Patients and Methods:}

Open randomized study comparing a series of patients treated with NSAIDs for 21 days and another who received steroid intraarticular infiltration (SIAI) spaced at every 7 days. The visual analog scale was used for the weekly assessment of spontaneous pain and pain when walking. Lequesne functional pain scale was used to assess the functional impact of knee osteoarthritis.

Results:

Seventy patients were enrolled, including 35 in the NSAID arm and 35 in SIAI arm. Forty-nine (70\%) had stage III of Kellgren and Lawrance scale. On admission, the average pain intensity was $50.46 \pm 30.93$ in the NSAID arm and $60.92 \pm 30$ in SIAI arm (p= $0.0189)$. At the end of follow-up, pain intensity was $6.72 \pm 13$ in NSAIDs patients and $17.80 \pm 21$ in SIAI one $(p=0.001)$. The average intensity of pain on walking was $64.41 \pm 22.61$. It was $53.33 \pm 22.31$ in NSAID's against $74.85 \pm 17.55$ in SIAI patients (P $<0.0001)$. At the end of the treatment, they were respectively $19.11 \pm 11.37$, and $35 \pm 30.69(\mathrm{P}=0.0085)$.

\section{Conclusion:}

Corticosteroid injections have a short efficacy compared to NSAIDs. Prescribing NSAIDs should consider the cons-indications, comorbidities and their deleterious digestive, renal, and cardiovascular effect.

Keywords: Cardiovascular effect, Knee osteoarthritis, NSAIDs, Steroid intra-articular injection.

\section{INTRODUCTION}

The non-steroidal anti-inflammatory drugs (NSAIDs) are known for their efficiency in the congestive osteoarthritis of the knee. Complications including cardiovascular were better documented in recent years and the indications most detailed [1].

\footnotetext{
* Address correspondence to this author at 09 BP 628 Ouagadougou. Burkina-Faso; Email: ouedd@yahoo.fr
} 
Steroid intra-articular infiltration (SIAI) is an effective alternative [2]; however, their effect seems short, not more than two to three weeks. In practice, the choice of treatment, NSAIDs or SIAI is not codified and predictors of efficacy of SIAI little known [3]. Few studies have compared the efficacy of NSAIDs and SIAI in short and medium terms in knee osteoarthritis.

The objective of this study was to compare the efficacy on pain and joint function of drug treatment with NSAIDs versus SIAI in knee osteoarthritis in congestive thrust.

\section{PATIENTS AND METHODS}

This was a prospective open randomized trial conducted from 15 July 2013 to 15 February 2014 in Rheumatology and Orthopedic Surgery department, at the University Hospital Yalgado Ouedraogo. Consenting patients coming in consultation for knee osteoarthritis in congestive thrust were included. Patients were openly randomized alternately in both parts and were monitored for 6 weeks either in consultation or by phone call. The patients who could not be monitored, changed treatment or associated other treatment were excluded from the study.

Table 1. Patient characteristics.

\begin{tabular}{|c|c|c|c|c|}
\hline & & NSAIDs & SIAI & Probability \\
\hline Average age (years) & & 59.68 & 46.40 & 0.005 \\
\hline Body mass index (kg/m2) & & $28.60 \pm 5$ & $30.21 \pm 4.93$ & 0.07 \\
\hline Topography of knee osteoarthritis & & & & \\
\hline & PF* & 2 & 2 & 0.12 \\
\hline & FT** & 14 & 5 & \\
\hline Overall & 19 & 28 & \\
\hline Kellgren and Lawrence classification & & & & \\
\hline & Stage 1 & 1 & 1 & 0.064 \\
\hline Stage 2 & 7 & 0 & \\
\hline Stage 3 & 25 & 24 & \\
\hline Stage 4 & 2 & & \\
\hline Affected knee & & & & \\
\hline & Right & 20 & & 10 \\
\hline
\end{tabular}

* Patello-femoral

** Femoral-tibial

Diclofenac and aceclofenac were used respectively at a dose of $150 \mathrm{mg}$ and $200 \mathrm{mg}$ twice daily for 21 days. The cortivazol 3,75 mg or betamethasone $2 \mathrm{mg}$ was used for intra-articular infiltration in a series of 3 injections spaced one week each. The algofunctional Lequesne index was assessed at the beginning and at the end of follow-up of each patient. The visual analog scale (0 to 100) was used to evaluate the intensity of pain whether spontaneous or when walking. If there is bilateral knee osteoarthritis, the most symptomatic knee was the target of the study.

The data were entered into computer and analyzed with the software Epi-Info version 3.5.1. Chi-square test and Fisher exact test were used to compare qualitative variables. The ANOVA test was used for comparison of means. The Statistical significance was $\alpha<0.05$. Oral and informed consent was required.

\section{RESULTS}

\section{Characteristics of the Target Population}

Eighty-three patients were included during the study period. During follow-up, 13 patients were excluded, five due to self medication and additional eight due to the ineffectiveness of treatment. Seventy patients were selected including 35 treated with NSAIDs and 35 who received SIAI. The average age of patients was 53.04 years with extremes of 32 and 78 years. These were 60 women $(85.7 \%)$ and ten men $(14.3 \%)$ or a sex ratio of 0.16 . Forty-nine patients $(70 \%)$ had grade III of Kellgren and Lawrence scale. The average algofunctional Lequesne index was 8.48. Table 1 shows the other patient characteristics.

\section{Assessment of Pain}

The intensity of spontaneous pain was $50.46 \pm 30.93$ in patients receiving NSAIDs and $63.92 \pm 30.07$ for those who 
received SIAI ( $p=0.0189$ ). Fig. (1) shows the evolution of the number of patients with spontaneous pain depending on the treatment.

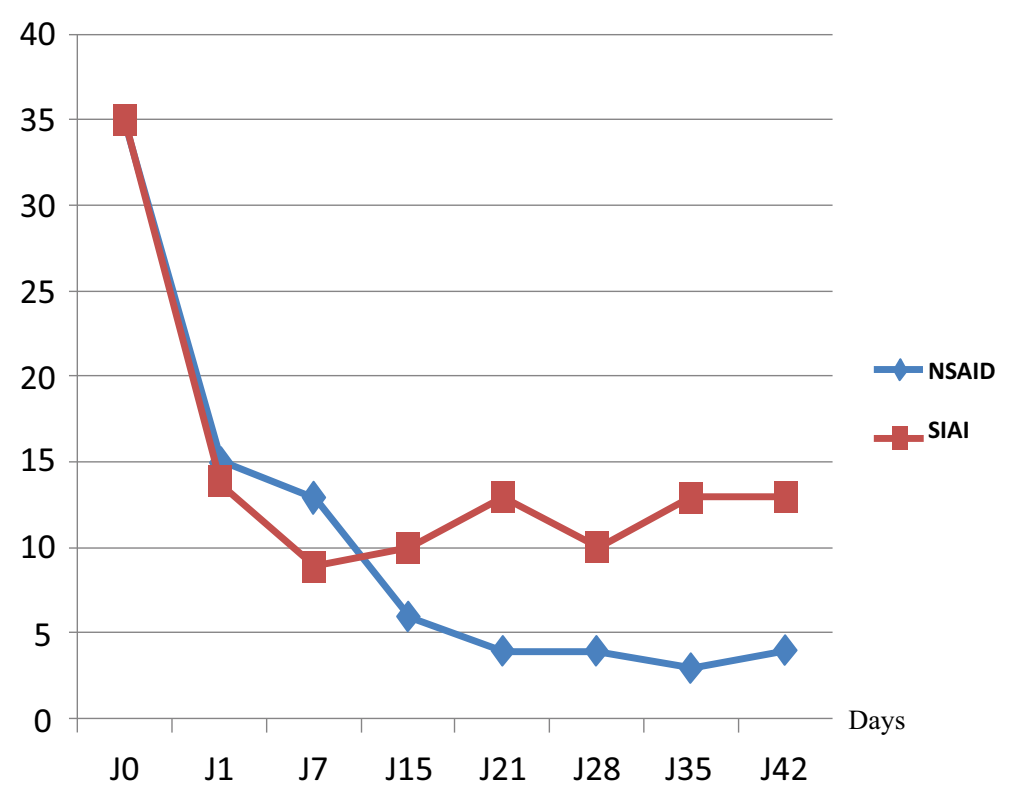

Fig. (1). Evolution of the number of patients with spontaneous pain depending on the type of treatment.

At the end of follow-up, pain intensity was $6.72 \pm 13.75$ in the population who received NSAIDs and $17.80 \pm 21.78$ in the one having obtained the SIAI $(p=0.00101)$. Fig. (2) shows the evolution of the intensity of spontaneous pain on processing. The average intensity of pain on walking was $64.41 \pm 22.61$. It was $53.33 \pm 22.31$ in NSAID against $74.85 \pm$ 17.55 in SIAI $(\mathrm{P}<0.0001)$. At the end of the treatment, they were respectively $19.11 \pm 11.37$, and $35 \pm 30.69(\mathrm{P}=$ 0.0085). Fig. (3) shows the change in the intensity of pain on walking after treatment.

Table 2 compares the effectiveness of NSAIDs $v s$. SIAI for different pain and the Lequesne index.

\section{Comments}

Few studies have compared the effectiveness of oral NSAIDs and SIAI in congestive knee osteoarthritis. Our study reports an efficiency of NSAIDs on steroid infiltration after a follow-up of 42 days.

Table 2. Comparative efficacy of NSAIDs versus SIAI on pain and Lequesne index at D0 and D42.

\begin{tabular}{|c|c|c|c|c|c|}
\hline & \multicolumn{2}{|c|}{ NSAIDs (n= 35) } & \multicolumn{2}{c|}{ SIAI (n = 35) } & Probability \\
\hline & D0 & $\mathbf{D 4 2}$ & D0 & D42 & \\
\hline VAS average & 50.5 & 6.7 & 63.9 & 17.8 & 0.0001 \\
\hline Spontaneous pain (\%) & 83 & 11.4 & 100 & 37.1 & 0.0012 \\
\hline Walking pain (\%) & 97 & 57 & 100 & 74.2 & 0.002 \\
\hline Pain when standing (\%) & 68.6 & 34.2 & 77.1 & 54.2 & 0.008 \\
\hline Prolonged standing pain (\%) & 71.4 & 25.7 & 91.4 & 51.4 & 0.0003 \\
\hline Means Lequesne index & 6.6 & 2.4 & 10.3 & 5.1 & 0.0001 \\
\hline
\end{tabular}

$\%=$ percentage of patients with pain

VAS: Visual Analog Scale

While the general patient characteristics are similar to other African series [4 - 6], there is difference between the two parts at inclusion which may constitute a bias; indeed, pain and functional impairment was higher in the part of patients with SIAI. Recently, a study of 32 patients with shoulder impingement syndrome showed the effectiveness of NSAIDs on the steroid injection; this efficiency is expressed in terms of gain in shoulder mobility assessed after one month of follow-up [7]. However, it seems to have no significant difference between the oral NSAIDs and steroid infiltration in treating carpal tunnel syndrome [8]. Instead, in the tendinitis of the shoulder and elbow, the efficacy of corticosteroid injections seems short [9]. This short-term efficacy also has been reported in knee osteoarthritis [10]. Indeed, from the seventh day of treatment, the number of patients with spontaneous pain in the NSAIDs part was 
significantly less than in patients who have received SIAI. Predictors of efficacy in the short and medium term of SIAI are not well known; however, the severity of the disease, the absence of synovitis in ultrasound, the presence of intraarticular effusion seem to lead to a more effective response [11,12]. Our series was characterized by the severity of knee osteoarthritis (Lequesne index to 8.48) and an intra articular effusion in all patients; the superiority of infiltration lasted only one week despite that two other infiltrations have been made. These results suggest that a combination of both treatments may be performed. This could make a single steroid infiltration on the first day followed by treatment of NSAID per os. This scheme would have the advantage of reducing the risk of infection that could be linked to the last two corticosteroid injections. Other studies, including the protocol to the two described here could better appreciate the superiority of a protocol on the other and tolerance in the patient.

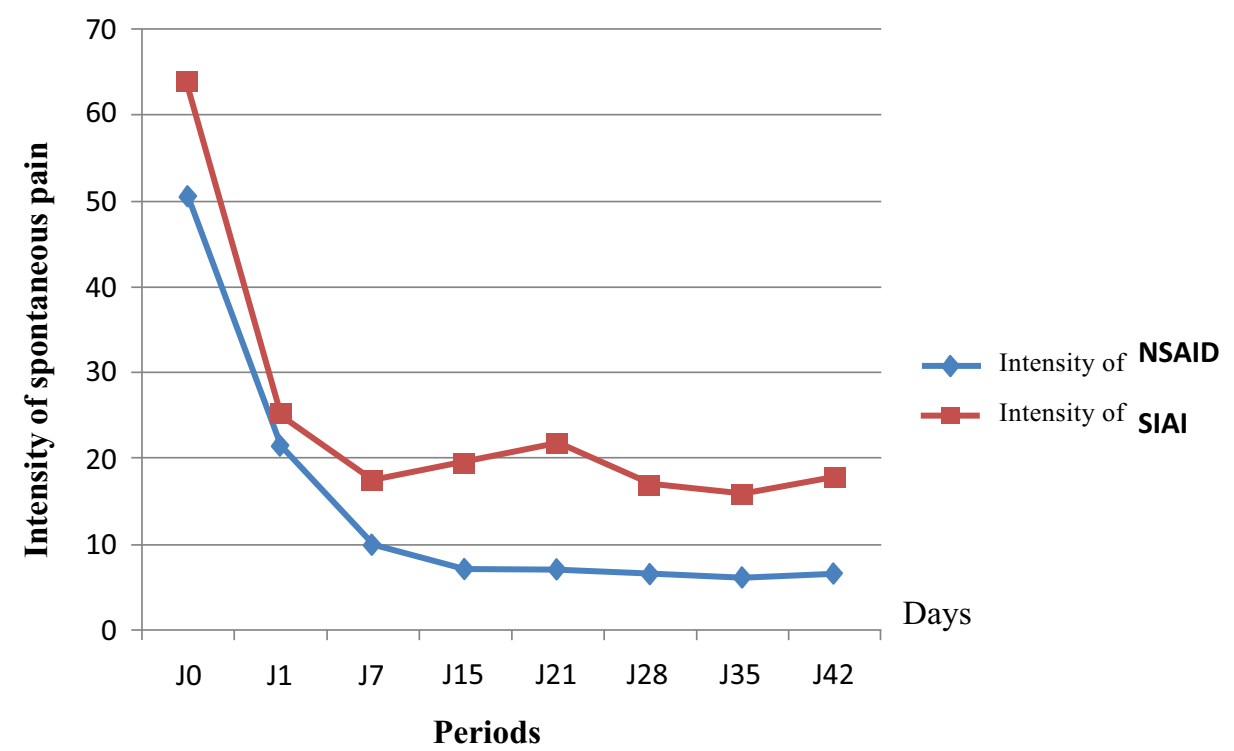

Fig. (2). Evolution of the intensity of spontaneous pain depending on the type of treatment.

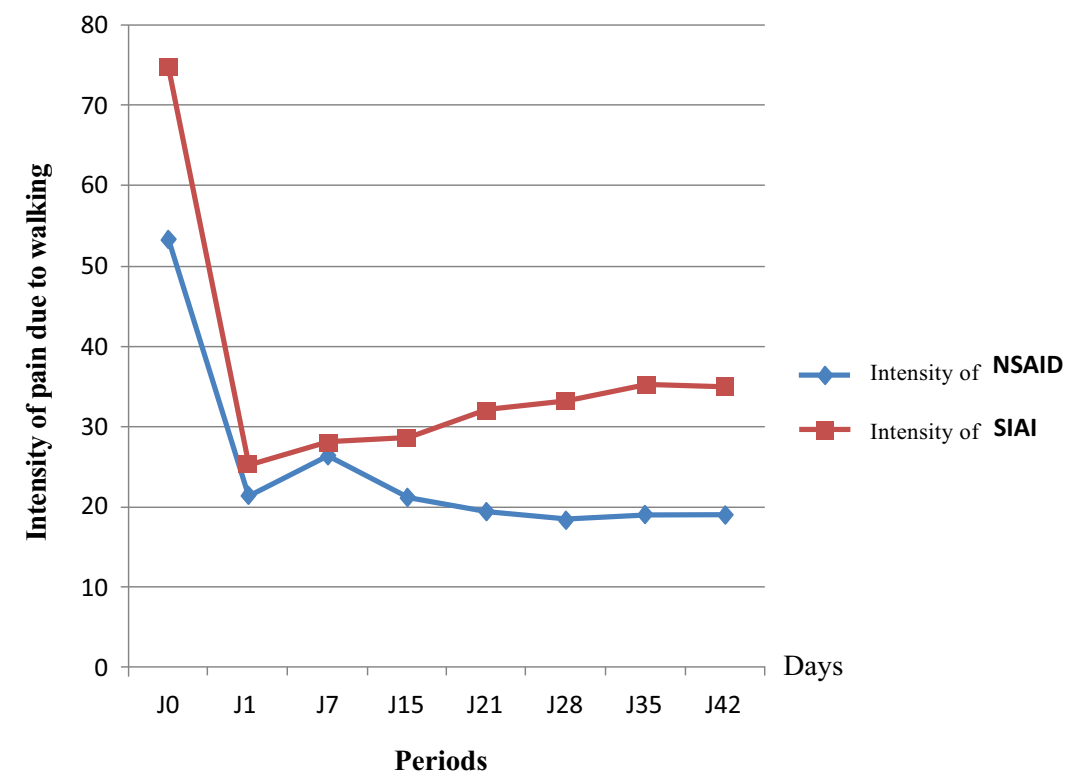

Fig. (3). Evolution of pain intensity due to walking according to the type of treatment.

\section{CONCLUSION}

SIAI seems to have a brief efficiency. The oral NSAIDs have the advantage of maintaining a relief in the period compared to steroid intra-articular infiltration. Their prescription should consider the contraindications and their 
deleterious effects including gastrointestinal, renal, and cardiovascular effects. The difference in the average ages of the 2 groups could be a limitation of the study

\section{CONFLICT OF INTEREST}

The authors confirm that this article content has no conflict of interest.

\section{ACKNOWLEDGEMENTS}

Declared none.

\section{REFERENCES}

[1] Bruyère O, Cooper C, Pelletier JP, et al. An algorithm recommendation for the management of knee osteoarthritis in Europe and internationally: a report from a task force of the European Society for Clinical and Economic Aspects of Osteoporosis and Osteoarthritis (ESCEO). Semin Arthritis Rheum 2014; 44(3): 253-63. [http://dx.doi.org/10.1016/j.semarthrit.2014.05.014] [PMID: 24953861]

[2] Konai MS, Vilar Furtado RN, Dos Santos MF, Natour J. Monoarticular corticosteroid injection versus systemic administration in the treatment of rheumatoid arthritis patients: a randomized double-blind controlled study. Clin Exp Rheumatol 2009; 27(2): $214-21$. [PMID: 19473560]

[3] Hirsch G, Kitas G, Klocke R. Intra-articular corticosteroid injection in osteoarthritis of the knee and hip: factors predicting pain relief--a systematic review. Semin Arthritis Rheum 2013; 42(5): 451-73.

[http://dx.doi.org/10.1016/j.semarthrit.2012.08.005] [PMID: 23374502]

[4] Mijiyawa M. Rheumatic diseases in black Africa. Rev Rhum Ed Fr 1993; 60(6): 451-7.

[PMID: 8124280]

[5] Eti E, Kouakou HB, Daboiko JC, et al. Epidemiology and features of knee osteoarthritis in the Ivory Coast. Rev Rhum Engl Ed 1998; 65(12): 766-70.

[PMID: 9923045]

[6] Ouédraogo DD, Séogo H, Cissé R, et al. Risk factors associated with osteoarthritis of the knee in a rheumatology outpatient clinic in Ouagadougou, Burkina Faso. Med Trop (Mars) 2008; 68(6): 597-9. [PMID: 19639826]

[7] Min KS, St Pierre P, Ryan PM, Marchant BG, Wilson CJ, Arrington ED. A double-blind randomized controlled trial comparing the effects of subacromial injection with corticosteroid versus NSAID in patients with shoulder impingement syndrome. J Shoulder Elbow Surg 2013; 22(5): 595-601.

[http://dx.doi.org/10.1016/j.jse.2012.08.026] [PMID: 23177167]

[8] Gurcay E, Unlu E, Gurcay AG, Tuncay R, Cakci A. Evaluation of the effect of local corticosteroid injection and anti-inflammatory medication in carpal tunnel syndrome. Scott Med J 2009; 54(1): 4-6.

[http://dx.doi.org/10.1258/rsmsmj.54.1.4] [PMID: 19291926]

[9] Gaujoux-Viala C, Dougados M, Gossec L. Efficacy and safety of steroid injections for shoulder and elbow tendonitis: a meta-analysis of randomised controlled trials. Ann Rheum Dis 2009; 68(12): 1843-9.

[http://dx.doi.org/10.1136/ard.2008.099572] [PMID: 19054817]

[10] Bellamy N, Campbell J, Robinson V, Gee T, Bourne R, Wells G. Intraarticular corticosteroid for treatment of osteoarthritis of the knee. Cochrane Database Syst Rev 2005; 18(2): CD005328. [PMID: 15846755]

[11] Maricar N, Callaghan MJ, Felson DT, O’Neill TW. Predictors of response to intra-articular steroid injections in knee osteoarthritis--a systematic review. Rheumatology (Oxford) 2013; 52(6): 1022-32.

[http://dx.doi.org/10.1093/rheumatology/kes368] [PMID: 23264554]

[12] Chao J, Wu C, Sun B, et al. Inflammatory characteristics on ultrasound predict poorer longterm response to intraarticular corticosteroid injections in knee osteoarthritis. J Rheumatol 2010; 37(3): 650-5. [http://dx.doi.org/10.3899/jrheum.090575] [PMID: 20080918]

Received: August 29, $2015 \quad$ Revised: November 5, 2015

Accepted: November 5, 2015

(C) Dieu-Donné et al.; Licensee Bentham Open.

This is an open access article licensed under the terms of the Creative Commons Attribution-Non-Commercial 4.0 International Public License (CC BY-NC 4.0) (https://creativecommons.org/licenses/by-nc/4.0/legalcode), which permits unrestricted, non-commercial use, distribution and reproduction in any medium, provided the work is properly cited. 